\title{
Caso clínico: Diagnóstico de alergia a relajantes musculares y opiáceos
}

\section{Case report: Diagnosis of allergy to muscle relaxants and opiates}

Ezequiel Amoroso', Mariana Monteiro', Ale Selva

\begin{abstract}
The anesthesiologist is the specialist who most often faces allergic reactions due to the number of drugs and substances that are exposed to the patient during the course of an intervention. Although they are rare, they have a mortality rate between $3 \%$ and $10 \%$. We present a clinical case of a 42 -year-old woman with a history of anaphylactic reaction grade 2 that is coordinated for a Werthein-Meigs surgery, for which it is decided to test her with skin tests. The results were positive for morphine, fentanil, remifentanil and atracurium, which directly affected the perioperative management.
\end{abstract}

\section{RESUMEN}

El anestesiólogo es el especialista que con mayor frecuencia se enfrenta a reacciones alérgicas por el número de fármacos y sustancias a las que se expone al paciente en el transcurso de una intervención. Si bien son poco frecuentes presentan una mortalidad entre 3 a 10\%. Presentamos un caso clínico de una mujer de 42 años con un antecedente de reaccion anafiláctica grado 2 que se coordina para una cirugía de Werthein- Meigs, por lo cual se decide testearla con pruebas cutáneas. Los rsultados fueron positivos para morfina, fentani, remifentanil y atracurio, lo cual incidió directamente en el manejo perioperatorio.

\section{Key words:}

Anphilaxis, muscle relaxants, opioids

\section{Palabras clave:}

Anafilaxia,

relajantes musculares, opiáceos

Hospital de Clínicas, Montevideo, Uruguay.

Fecha de ingreso: 05 de diciembre de 2018

Fecha de aceptación: 29 de diciembre de 2018

\section{ORCID}

https://orcid.org/0000-0002-1243-2615

Correspondencia:

Mariana Monteiro

E-mail: monteirocmariana@gmail.com 


\section{Introducción}

as reacciones anafilácticas alérgicas y no alérgicas severas que ocurren durante la intervención quirúrgica son de las mayores preocupaciones para el anestesiólogo. Tienen una incidencia que varía desde $1 / 3.500$ a 1/20.000 anestesias, según el país estudiado[1],[2], vinculado probablemente a un subregistro de los casos.

Las reacciones anafilácticas son poco frecuentes pero potencialmente fatales, con una mortalidad estimada entre $3-10 \%$ y daño neurológico significativo hasta en el $2 \%$ de los casos[3]. A pesar de esta evidencia, son muchos los pacientes que llegan al preoperatorio inmediato sin un adecuada valoración ni preparación previas.

La importancia de conocer este diagnóstico radica en que se pueden implementar medidas tales como los tests cutáneos, evaluando fármacos a utilizar con seguridad, preparación preoperatoria y estrecha vigilancia durante todo el perioperatorio. Planteamos un caso clínico en el que se realiza diagnóstico imprevisto de reacción anafiláctica en el preoperatorio inmediato, repercutiendo directamente en la oportunidad operatoria, preparación previa a la cirugía y planificación de técnica anestésica y analgésica.

\section{Caso clínico}

Paciente de sexo femenino, 42 años, coordinada para Wertheim Meigs. AAQ: polipectomía de cuerdas vocales a los 20 años con anestesia general sin incidentes. Hace 5 meses anestesia general para resección de otro pólipo en la que refiere rush cutáneo, edema generalizado y broncoespasmo, realizada en otro centro hospitalario.

No se realizaron estudios ni tratamiento luego de este episodio.

Como antecedentes alérgicos refiere reacciones cutáneas de tipo máculo-pápula frente a picaduras de insectos.

No fue pesquisada en la consulta preoperatoria alejada. Dado que en visita médica previa al ingreso a block quirúrgico se clasificó esta reacción como grado dos en la escala de severidad[2],[3] se decide estudiarla rápidamente, sin diferir la intervención más de lo necesario por ser una cirugía oncológica. Se realiza prick test e intradermorreacción. Se detallan en el siguiente cuadro los fármacos testados, las diluciones utilizadas y los resultados.

Durante la prueba se constata dermatografía (Figura 1) por lo que los resultados se compararon con suero fisiológico e histamina como soluciones de control. Como se evidencia en Tabla 1 fueron detectadas reacciones cutáneas positivas a: morfina, fentanil, remifentanil y atracurio.

Se premedicó a la paciente con ranitidina, fexofenadina y prednisona una semana previa a la cirugía. Los resultados condicionaron la técnica anestésica seleccionada que consistió en realizar anestesia peridural en base a anestésicos locales únicamente y mantener el catéter para analgesia postoperatoria. La intervención duró 4 horas y transcurrió sin incidentes, fue bien tolerada por la paciente y no hubo inconvenientes para el equipo quirúrgico. Se mantuvo la infusión en base a bupivacaína al 0,125\% por 72 horas y luego se rotó a anlagésicos intravenosos libres de opiáceos. Se otorgó el alta a domicilio a la semana sin inconvenientes.

\section{Discusión}

La paciente presenta el antecedente de una reacción previa relacionada con la anestesia de grado

\begin{tabular}{ll} 
& Tabla $\mathbf{1}$ \\
\hline Prick test & Intradermorreacción \\
Propofol (puro): negativo & Propofol (1:100): negativo \\
Fentanyl (puro): negativo & Fentanyl (1:10): positivo \\
Atracurio (1:10): negativo & Atracurio (1:1000): positivo \\
Succinilcolina (1:5): negativo & Succinilcolina (1:500): negativo \\
Morfina (1:10): positivo & \\
Rocuronio (puro): negativo & Rocuronio (1:100): negativo \\
Remifentanyl (puro): negativo & Remifentanyl (1:100): positivo \\
Tramadol (puro): negativo & Tramadol (1:1.000): negativo \\
\hline
\end{tabular}




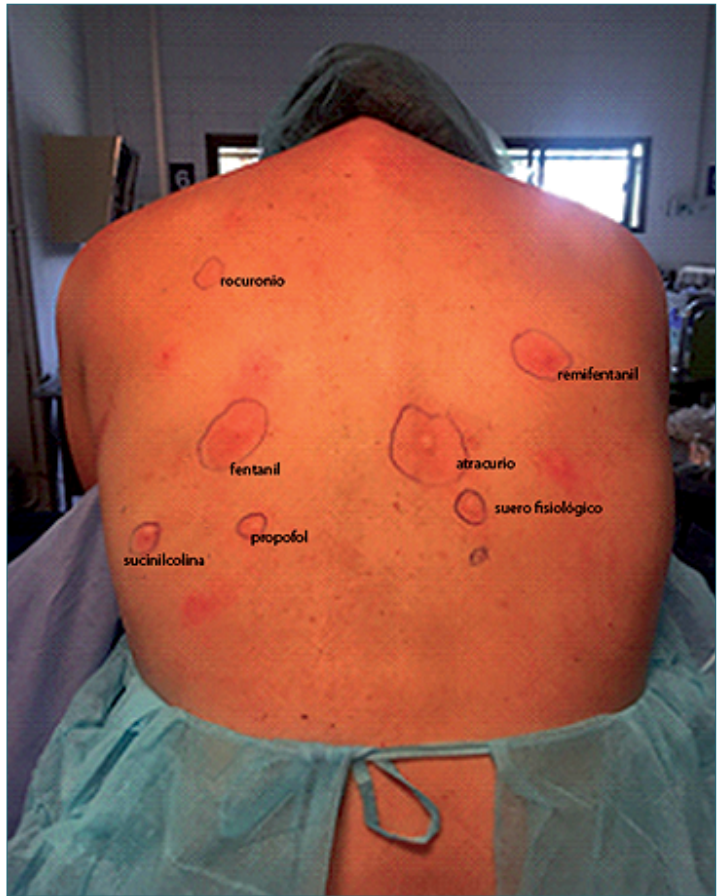

Figura 1. Intradermorreacción. Respuesta positiva a atracurio, fentanil y remifentanil.

2 dada por rush cutáneo y broncoespasmo[2], por lo cual tenía indicación formal de ser estudiada con test cutáneos en el preoperatorio alejado.

Debemos resaltar la importancia de la valoración clínica preanestésica relacionada con la alergia realizada por el anestesiólogo como una oportunidad para la pesquisa de estas reacciones y el registro en la historia clínica de las mismas.

En nuestro medio la valoración alérgica se basa en test cutáneos. No contamos con centros de alergoanestesiología ni disponemos de dosificación plasmática de triptasa y pruebas in vitro de IgE específicas, limitando nuestro diagnóstico.

No obstante, las pruebas cutáneas no requieren de material sofisticado para su ejecución y las drogas testeadas son aquellas disponibles para la práctica diaria.

En nuestro caso los resultados de las pruebas realizadas modificaron la técnica anestésica y analgésica y permitieron implementar un tratamiento profiláctico en base a antihistamínicos y corticoides, medidas que han demostrado impactar directamente en una reducción de la morbimortalidad de la paciente[4],[5].
Se recomienda la valoración de las pruebas en unidades multidisciplinares de referencia con experiencia en la realización e interpretación de las mismas. Se deben estudiar todos los fármacos administrados inmediatamente antes de la reacción de hipersensibilidad. El valor predictivo del prick test varia según la droga estudiada. En la mayoría de los casos una prueba positiva hace que la reacción anafiláctica sea muy probable, mientras que un resultado negativo no indica necesariamente que el paciente no tendrá alguna reacción de hipersensibilidad[6]. La sensibilidad de las pruebas cutáneas para los relajantes neuromuculares es de hasta $97 \%$. La sensibilidad es también alta para otras sustancias tales como látex, $\beta$-lactámicos, propofol y disminuye para opioides, benzodiacepinas, AINE, contrastes radiológicos y clorhexidina. No se recomienda la realización con opiáceos como la morfina, codeína, o meperidina, pues son fármacos activadores del mastocito incurriendo habitualmente en reacciones falsamente positivas. Sin embargo, son útiles si los agentes implicados son los opioides sintéticos (fentanilo, remifentanilo). Los relajantes neuromusculares tienen una reactividad cruzada del $60-80 \%$ por el amonio cuaternario que comparten; por ello cuando las pruebas cutáneas son positivas a uno estamos obligados a estudiar el resto de los relajantes hasta encontrar alguno negativo. Las pruebas intradérmicas deben ser realizadas cuando los prick test son negativos, teniendo en cuenta que son más sensibles pero menos específicas, más difíciles de interpretar y pueden provocar una reacción sistémica ${ }^{7}$.

En el último estudio retrospectivo realizado en Francia por la GERAP en enero de 2011 a diciembre de 2012 se encontró que el $68,5 \%$ de las reacciones en anestesia eran de tipo alérgico mediadas por IgE y el $31,5 \%$ eran no inmunomediadas.

Todas las drogas y sustancias usadas durante la cirugía y la anestesia, tal vez con la única excepción de los agentes inhalatorios, han sido reportadas como potencialmente causantes de reacciones anafilácticas. Coincidente con la revisión de la GERAP nuestra paciente tuvo una respuesta positiva a relajantes musculares no despolarizantes, siendo positiva a atracurio, cuya incidencia es de 13,9\%. Estas reacciones son inmunomediadas por lgE[8].

Por otra parte, las reacciones de hipersensibilidad a los opioides son raras y la mayoría se deben a reacciones no alérgicas por liberación de histamina, aunque existen casos aislados de anafilaxia mediada por IgE principalmente en opiáceos de alta potencia (fentanil e hidromorfona)[9]. Baldo y Pham consideran que las reacciones adversas a los opioides, más que debidas a liberación de histamina, son reacciones de 
tipo no alérgico con predominio de signos cutáneos más que pulmonares, y que la hipotensión, cuando se presenta, es probablemente debida a reacciones alérgicas[10],[11]. En esta paciente, tuvimos pruebas cutáneas positivas para opioides.

Los corticoides y los anti- H1 han demostrado reducir la incidencia e intensidad de las reacciones no mediadas inmunológicamente, pero no son eficaces previniendo las reacciones alérgicas, si bien podrían disminuir los efectos de la histamina al estar bloqueando sus receptores[4].

A pesar que la técnica combinada general y peridural es la más utilizada en este cirugía, la evidencia muestra que la única medida eficaz para evitar las reacciones anafilácticas es evitar el contacto con el agente desencadenante, teniendo en cuenta que si bien sensibilidad de las pruebas cutáneas es alta, no llega al $100 \%$.

\section{Conclusión}

Presentamos el caso de una paciente que presentó pruebas cutáneas positivas a relajantes musculares y opioides y se planteó como alternativa realizar anestesia peridural en base a anestésicos locales, que se llevó a cabo con éxito, permaneciendo con analgesia peridural en el postoperatorio.

El anestesiólogo es el especialista que con mayor frecuencia se enfrenta a reacciones alérgicas por el número de fármacos y sustancias a las que se expone al paciente en el transcurso de una intervención. Destacamos la importancia de detectar estos pacientes en el preparatorio y estudiarlos previamente. Asimismo, la detección temprana en el preparatorio permite el tratamiento previo en base a corticoides y antihistamínicos que han demostrado disminuir la severidad de las de las reacciones[3].

\section{Referencias}

1. Criado A, Seiz A, Ortiz JR. Reacciones alérgicas en anestesia. Servicio de Anestesiología y Reanimación. http://www.hvil.sld. cu/bvs/archivos/2_cap_02\%20 reacciones \%20alergicas.pdf

2. Mertes PM, Laxenaire MC. Allergic reactions occurring during anaesthesia. Eur J Anaesthesiol. 2002 Apr;19(4):240-62. https:// doi.org/10.1097/00003643200204000-00002 PMID:12074414

3. Bustamante RB, Luxoro CV, et al. Anafilaxia perioperatoria: cuadro clínico y diagnóstico. Rev Chil Anest. 2010;39:36-52.

4. Mertes PM, Malinovsky JM, Jouffroy L, Aberer W, Terreehorst I, Brockow K, et al.; Working Group of the SFAR and SFA; ENDA; EAACI Interest Group on Drug Allergy. Reducing the risk of anaphylaxis during anesthesia: 2011 updated guidelines for clinical practice. J Investig Allergol Clin Immunol. 2011;21(6):44253. PMID:21995177

5. Kroigaard M, Garvey LH, Gillberg
L, Johansson SG, Mosbech $\mathrm{H}$, Florvaag $\mathrm{E}$, et al. Scandinavian Clinical Practice Guidelines on the diagnosis, management and follow-up of anaphylaxis during anaesthesia. Acta Anaesthesiol Scand. 2007 Jul;51(6):65570. https://doi.org/10.1111/ j.1399-6576.2007.01313.x PMID:17567266

6. Heinzerling $L$, Mari $A$, Bergmann KC, Bresciani M, Burbach G, Darsow $U$, et al. The skin prick test - European standards. Clin Transl Allergy. 2013 Feb;3(1):3. https:// doi.org/10.1186/2045-7022-3-3 PMID:23369181

7. Escolano Villen F, Sanchez Navas S, Yelamos López J, Espona Quer M, Giménez Arnau A, Sierra Arnedo P. Guía de actuación de la anafilaxia en anestesia. Mayo 2016. Disponible en http://www. scartd.org/sap/docs/Anafilaxia. pdf

8. Tacquard C, Collange O, Gomis $P$, Malinovsky JM, Petitpain N, Demoly $\mathrm{P}$, et al. Anaesthetic hypersensitivity reactions in France between 2011 and 2012: the 10th GERAP epidemiologic survey. Acta Anaesthesiol Scand. 2017 Mar;61(3):290-9. https:// doi.org/10.1111/aas.12855 PMID:28164269

9. Montañez MI, Mayorga C, Bogas G, Barrionuevo E, FernándezSantamaria R, Martin-Serrano A, et al. Epidemiology, mecanismos and diagnosis of drug- inducen anaphilaxys. Front Immunol. 2017 May;8:614. https://doi. org/10.3389/fimmu.2017.00614 PMID:28611774

10. Baldo BA, Pham NH. Histamine-releasing and allergenic properties of opioid analgesic drugs: resolving the two. Anaesth Intensive Care. 2012 Mar;40(2):216-35. https://doi.g/ 10.1177/0310057X1204000204 PMID:22417016

11. Aguilera-Castro F. Reincidencia intraoperatoria de probable reacción alérgica al remifentanilo. Informe de caso. Revista Colombiana de Anestesiología. Ovid Technologies (Wolters Kluwer Health); 2017 Jan;45:31-5. Disponible en: http://dx.doi.org/10.1016/j. rca.2016.04.007 